\title{
Modificación de las residencias de Anestesiología durante la pandemia en la Argentina
}

\section{Anesthesiology residencies modifications during Argentina's COVID-19 pandemia}

Facundo José Balderrama MD. ${ }^{1}$, Roberta Inés Ladenheim Mg. ${ }^{2}$, Marcelo Machain MD. ${ }^{3}$, Hernán Miguel Montenegro MD. ${ }^{4}$, Claudio Alberto Tartaglia Pulcini MD. ${ }^{5}$, Hugo Daniel Gutiérrez MD. ${ }^{6}$

\begin{abstract}
Background and Objective: Residents education has been affected worldwide by changes brought about at both educational and healthcare system levels. Programs in anesthesiology have been impacted by changes in health services and the need for social isolation. This paper is aimed at describing changes in anesthesiology residencies resulting from the COVID-19 pandemic Material and Methods: An anonymous survey was conducted in order to explore anesthesiology residents' perception in the Republic of Argentina re. changes and adaptations in study programs and their emotional impact. Results: 427 out of 810 residents (52.7\% answering rate) from all over the country answered the survey. 14\% caught COVID-19 and around half of them had to self-isolate. $78.2 \%$ of residents reported their studies were affected during the pandemia, $79 \%$ agreed on a decrease in the number of procedures, $72.1 \%$ in scheduled rotations, and $67.4 \%$ in attending classes. Residents consistently stated increased stress, episodes of anxiety, sadness and depression as well as sleep disorders or difficulties. No reference was made to an increase in the intake of alcohol or other substances. Conclusion: The development of anesthesiology learning programs as well as personal lives of residents have been negatively affected by the pandemia.
\end{abstract}

\section{Key words:}

Medical education, COVID-19, anesthesiology, residencies

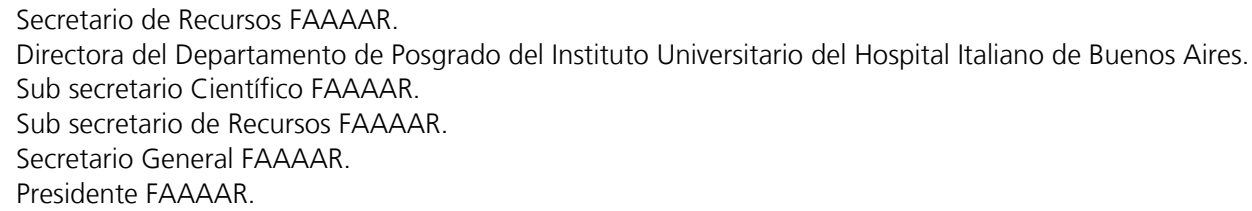

Fecha de ingreso: 03 de diciembre de 2020

Fecha de aceptación: 12 de diciembre de 2020

\section{ORCID}

0000-0002-0088-6951

\section{Correspondencia:}

Roberta Inés Ladenheim

roberta.ladenheim@hospitalitaliano.org.ar 


\section{RESUMEN}

Antecedentes y objetivo: La formación de los residentes del mundo se vio afectada por los cambios que implicó la pandemia a nivel de los sistemas educativos y de salud. El desarrollo de los programas de formación de anestesiología se vio impactado por las modificaciones de las prácticas en los servicios de salud y la necesidad de aislamiento social. El objetivo de este trabajo fue describir los cambios en la formación de residencias de anestesiología producidos a partir de la pandemia COVID-19. Materiales y Métodos: Se desarrolló una encuesta para indagar anónimamente la percepción de los residentes de anestesiología de la República argentina sobre los cambios y adaptaciones en los programas de formación y su afectación emocional. Resultados: Respondieron la encuesta 427 un total de 810 residentes (tasa de respuesta 52,7\%), provenientes de todo el país. El $14 \%$ se contagió de COVID-19 y cerca de la mitad debió realizar aislamiento. El 78,2\% de los residentes refirió que su formación se vio afectada durante la pandemia, $79 \%$ coincidió en el descenso del número de procedimientos, $72,1 \%$ en el descenso de las rotaciones programáticas y $67,4 \%$ en la disminución de las clases presenciales. Los residentes coinciden en haberse sentido más estresados, experimentado episodios de ansiedad, tristeza y depresión, y haber tenido trastornos del sueño o dificultades para dormir. No refieren haber aumentado el consumo de alcohol u otras sustancias. Conclusión: La pandemia afectó negativamente el desarrollo de los programas de formación de anestesiología y la vida personal de los residentes de anestesiología.

\section{Palabras clave:}

Educación médica, COVID-19, anestesiología, residencias

\section{Introducción}

L

a pandemia ha tenido un impacto en el funcionamiento de todos estratos de la sociedad de diferente manera. Han sido particularmente afectados tanto el sistema de salud, como el sistema educativo. En este contexto, las formaciones de posgrado que involucran la capacitación en servicio como son las residencias médicas, presentan particularidades debido al riesgo potencial para pacientes y profesionales en formación; debiendo adaptar los currículos a las nuevas necesidades y posibilidades asistenciales[1],[2].

Diversos estudios describen las modificaciones en el desarrollo de las residencias, tanto en lo que respecta la vulnerabilidad de los residentes al contagio, los cambios en los programas de formación, la pérdida de oportunidades de aprendizaje, la alteración del número de guardias, de la supervisión y bienestar de los residentes, entre otros[3]. Muchos residentes se han dedicado a tareas distintas a las enunciadas en el plan de estudios de su especialidad[4], requiriendo entrenamiento en el uso de equipos de protección personal (EPP), en el manejo de protocolos específicos para los pacientes con sospecha o confirmación de infección por COVID-19 y monitoreo de estos pacientes. Los residentes se han visto afectados tanto al enfermarse por COVID-19 como, psicológicamente al verse enfrentados a situaciones complejas[5]. Muchas veces los programas de formación no disponen de dispositivos adecuados para contener estas situaciones. Sus oportunidades de aprendizaje se han visto modificadas, con suspensión de procedimientos electivos habituales y cambios en los ámbitos de práctica, reducción de las patologías y suspensión de rotaciones. Todas estas modificaciones deben ser reconocidas para una planificación que contemple la recuperación de aprendizajes perdidos. Muchas de las actividades académicas de los programas debieron ser trasladas a la virtualidad, con suspensión de algunas que requerían presencialidad y otras se postergaron debiendo rediseñar la oferta formativa académica.

Las residencias de las diversas especialidades se vieron afectadas de manera distinta[6],[7], las especialidades que se ocupan de la atención de pacientes infectados con COVID-19 como clínica médica, emergentología, infectología y terapia intensiva han visto fuertemente incrementadas sus tareas. Otras especialidades han reorientado sus actividades para apoyar a aquellas más fuertemente comprometidas con la atención de pacientes afectados por la pandemia. Las residencias de anestesiología en el mundo[8],[9] han visto interrumpidas sus formaciones por las reorganizacio- 
nes de los servicios; con impacto en las rotaciones, la reducción del número de prácticas y procedimientos y la suspensión de las actividades académicas presenciales; viéndose afectado el desarrollo de los programas y las evaluaciones. La disminución en el número de procedimientos electivos condicionó las posibilidades de aprendizaje[10], especialmente aquellos que involucran la manipulación de la vía aérea implica alto riesgo de contagio[11. Los residentes y docentes han tenido que adaptarse a estas nuevas circunstancias, muchas veces utilizando medios digitales para sostener las actividades educativas. Diversas readaptaciones intentan mitigar estas dificultades en la formación[12].

Se han publicado varias experiencias que describen cómo los diferentes sistemas de residencias de diversos contextos han intentado adaptar sus procesos de aprendizaje y actividades asistenciales. No hemos encontrado en nuestro medio estudios que describan las actividades desarrolladas por residentes de anestesiología. Por lo tanto, nuestro objetivo fue describir las actividades desarrolladas por los residentes de anestesiología y evaluar cómo se afectaron sus actividades de aprendizaje durante la pandemia.

\section{Objetivo general}

Describir los cambios en la formación de residencias de anestesiología producidos a partir de la pandemia COVID-19.

\section{Objetivos específicos}

Describir cambios o adaptaciones en los programas de formación.

Describir cambios en las actividades prácticas.

Describir la percepción de afectación emocional por parte de los residentes.

\section{Material y Métodos}

Se diseñó un estudio de corte transversal. La población fueron todos los médicos residentes de anestesiología de la República Argentina. El protocolo fue aprobado por el Comité de Ética y Protocolos de Investigación del Instituto Universitario del Hospital Italiano de Buenos Aires (Proyecto N 0024-20).

\section{Población}

Se invitó a participar a todos los residentes de anestesiología a través de un correo electrónico con un enlace que conducía a una encuesta electrónica anónima y confidencial. Se realizaron dos recordatorios con intervalos de una semana. El estudio se desarrolló durante 14 días consecutivos en las semanas epidemiológicas 39 y 40.

\section{Diseño del instrumento}

Se diseñó una encuesta semiestructurada. El instrumento se construyó a partir de una revisión de bibliografía y una exploración a través de consultas a distancia a 7 docentes y 9 residentes de programas de anestesia de la República Argentina. Dos autores de forma independiente revisaron las respuestas y codificaron las mismas, luego acordaron codificaciones de temas en forma conjunta. La exploración exploró las siguientes tres preguntas: ¿qué aspectos de los programas de residencias piensa que se afectaron durante la pandemia?; ¿qué competencias, contenidos, habilidades o destrezas considera que no pudieron desarrollarse en este período? y ¿qué ganancias en la formación de los residentes tuvo la pandemia? Una vez identificados los temas, se definieron los ítems para cada tema: cinco ítems indagaron datos demográficos, nueve ítems los aspectos del programa que se modificaron a partir de la pandemia, cinco ítems la organización de las instituciones (capacitación específica para COVID-19, existencia de protocolos, acompañamiento), nueve ítems las actividades realizadas por los residentes vinculadas a COVID-19 y el contagio y la necesidad de aislamiento, y ocho ítems la afectación sobre el estado de ánimo y el bienestar. Las opciones de respuesta se organizaron en opciones de frecuencia (disminuyó, se mantuvo igual, aumentó o nunca hubo) y en una escala de Likert de cinco opciones de grado de acuerdo (1: totalmente en desacuerdo; 2: en desacuerdo; 3: ni de acuerdo ni en desacuerdo; 4: de acuerdo; 5: totalmente de acuerdo). Se preguntó con opciones de respuesta abierta acerca de las preocupaciones, aspectos negativos y positivos de la pandemia. La aceptabilidad y comprensión de la encuesta final fue corroborada con una muestra piloto de siete residentes y cinco anestesiólogos vinculados a la formación en residencias, luego de la cual se realizaron ajustes a la misma (Anexo 1).

\section{Análisis de datos}

Las variables cuantitativas se describieron por su media y desvío estándar y las categóricas en números absolutos y frecuencias. La escala de likert de acuerdo 
se expresó en media y desvío estándar. Se realizó la prueba de ji al cuadrado y t de Student para identificar asociaciones entre variables categóricas y continuas respectivamente. Un valor de p menor a 0,05 se consideró significativo. Se utilizó el programa estadístico Stata en su versión 15. Para el análisis cualitativo se definieron categorías luego de la lectura de los textos por dos investigadores en forma independiente, posteriormente, se consensuaron las categorías entre los investigadores y se seleccionaron verbatims representativos de cada una.

\section{Resultados}

Se obtuvieron 427 respuestas sobre un total de 810 residentes (tasa de respuesta 52,7\%). Los residentes que respondieron la encuesta presentaron una edad media de 30,1 años, pertenecían al género masculino en $54,7 \%$ y pertenecían a todos los años de la residencia.

El 50\% desarrollaron sus programas en la provincia de Buenos Aires y Ciudad de Buenos Aires y realizaron su formación en establecimientos de gestión pública en el 90,4\% como se describe en la Tabla 1. Respondieron de 18 provincias o distritos diferente, la procedencia de los respondedores fue Buenos Aires 137 (32\%), CABA 76 (17,8\%), Córdoba 35 (8,2\%), Mendoza 35 (8,2\%), Entre Ríos 22 (5,1\%), Chaco 19 (4,4\%), Neuquén $16(3,7 \%)$, Salta $15(3,5 \%)$, Santiago del Estero 12 (2,8\%), Jujuy 11 (2,5\%), Río Negro $11(2,5 \%)$, Formosa 10 (2,3\%), Santa Fé 9 (2,1\%), Tucumán $8(1,8 \%)$, Misiones $5(1,1 \%)$, Corrientes 3 $(0,7 \%)$, Santa Cruz 2 (0,4\%), La Rioja $1(0,2 \%)$.

La mayoría refirió que se organizaron equipos de trabajo para las tareas asistenciales y que debió asistir pacientes con COVID-19. El 14\% se contagió como producto de su práctica profesional, cerca de la mitad debió realizar aislamiento y $72 \%$ tuvo compañeros enfermos por COVID-19. La mayoría refirió que su formación se vio afectada durante la pandemia (Tabla 2). La percepción que la formación fue afectada por la pandemia se asoció al tipo de gestión de la residencia $81 \%$ pública vs 53,5 privada $(p<0,001)$ y año de ingreso a la residencia $16 \%$ en 2016 vs 30,7\% en 2017 vs 29,5 en 2018 vs 23,2\% en 2019 ( $p=0,013$ ). No se asoció al género ni edad de los residentes.

Con respecto a las características de las actividades educativas y asistenciales durante la pandemia, los resultados mostraron que en la mitad de los casos el número de guardias se mantuvo y aumentó sólo en 16,8\%. El 79\% coincidió en el descenso en el número de procedimientos y $72,1 \%$ en el descenso de las rotaciones programáticas. Las clases presenciales disminuyeron en $67,4 \%$ y las actividades de simulación en la mitad de los encuestados. El 30\% refirió disminución de la supervisión clínica, si bien en $60 \%$ de las respuestas la misma se mantuvo igual a previo a la pandemia. Se mantuvieron las estrategias de evaluación en el 67,6\%. El número de anestesiólogos por quirófano se mantuvo igual en $48 \%$, pero disminuyó el número de residentes por quirófano (Tabla 3).

Los residentes refirieron que en sus sedes se desarrollaron protocolos COVID-19 en las siguientes espacios asistenciales y educativos: quirófanos 411 $(96,2 \%)$, sala de internación $261(61,1 \%)$, consultorios $238(55,4 \%)$, y en menor medida en comedores 204 (47,8\%), aulas y/o espacios para actividades académicas $122(28,5)$. Doce residentes $(2,8 \%)$ refirieron que no se instalaron protocolos en ningún espacio.

Los encuestados coincidieron en que recibieron información periódica sobre la pandemia y entrenamiento en el uso de EPP y protocolos, y se sintieron capacitados para asistir pacientes COVID-19 y para

\begin{tabular}{llcr}
\hline & Tabla 1. Características de los profesionales & y sus espacios de formación \\
\hline Edad & media, DS & $\mathbf{3 0 , 1} \mathbf{3 , 3}$ & \% \\
& & $\mathbf{n}$ & 44,2 \\
Género & Femenino & 189 & 54,7 \\
& Masculino & 233 & 0,4 \\
& Otro & 2 & 0,7 \\
Año de ingreso & Prefiero no contestar & 3 & 16,6 \\
& 2016 & 71 & 27,8 \\
& 2017 & 119 & 29,0 \\
Tipo de Gestión & 2018 & 124 & 26,4 \\
& 2019 & 113 & 90,4 \\
\hline
\end{tabular}




\begin{tabular}{|c|c|c|c|}
\hline & & $\mathbf{n}$ & $\%$ \\
\hline Número de Guardias & $\begin{array}{l}\text { Disminuyó } \\
\text { Se mantuvo igual } \\
\text { Aumentó } \\
\text { Nunca hubo }\end{array}$ & $\begin{array}{r}125 \\
228 \\
72 \\
2\end{array}$ & $\begin{array}{r}29,2 \\
53,4 \\
16,8 \\
0,4\end{array}$ \\
\hline Descanso de Guardias & $\begin{array}{l}\text { Disminuyó } \\
\text { Se mantuvo igual } \\
\text { Aumentó } \\
\text { Nunca hubo }\end{array}$ & $\begin{array}{r}53 \\
190 \\
125 \\
59\end{array}$ & $\begin{array}{r}12,4 \\
44,5 \\
29,2 \\
1,8\end{array}$ \\
\hline Número de Procedimientos & $\begin{array}{l}\text { Disminuyó } \\
\text { Se mantuvo igual } \\
\text { Aumentó } \\
\text { Nunca hubo }\end{array}$ & $\begin{array}{r}339 \\
72 \\
15 \\
1\end{array}$ & $\begin{array}{r}79,3 \\
16,8 \\
3,5 \\
0,2\end{array}$ \\
\hline Clases Presenciales & $\begin{array}{l}\text { Disminuyó } \\
\text { Se mantuvo igual } \\
\text { Aumentó } \\
\text { Nunca hubo }\end{array}$ & $\begin{array}{r}288 \\
42 \\
15 \\
82\end{array}$ & $\begin{array}{r}67,4 \\
9,8 \\
3,5 \\
19,2\end{array}$ \\
\hline Actividades de Simulación & $\begin{array}{l}\text { Disminuyó } \\
\text { Se mantuvo igual } \\
\text { Aumentó } \\
\text { Nunca hubo }\end{array}$ & $\begin{array}{r}217 \\
28 \\
10 \\
172\end{array}$ & $\begin{array}{r}50,8 \\
6,5 \\
2,4 \\
40,2\end{array}$ \\
\hline Consultas de Telemedicina & $\begin{array}{l}\text { Disminuyó } \\
\text { Se mantuvo igual } \\
\text { Aumentó } \\
\text { Nunca hubo }\end{array}$ & $\begin{array}{r}55 \\
56 \\
110 \\
206\end{array}$ & $\begin{array}{l}12,8 \\
13,1 \\
25,7 \\
48,2\end{array}$ \\
\hline Rotaciones Programáticas & $\begin{array}{l}\text { Disminuyó } \\
\text { Se mantuvo igual } \\
\text { Aumentó } \\
\text { Nunca hubo }\end{array}$ & $\begin{array}{r}308 \\
29 \\
1 \\
89\end{array}$ & $\begin{array}{r}72,1 \\
6,7 \\
0,2 \\
20,8\end{array}$ \\
\hline Supervisión Clínica & $\begin{array}{l}\text { Disminuyó } \\
\text { Se mantuvo igual } \\
\text { Aumentó } \\
\text { Nunca hubo }\end{array}$ & $\begin{array}{r}133 \\
259 \\
12 \\
23\end{array}$ & $\begin{array}{r}31,1 \\
60,6 \\
2,8 \\
5,3\end{array}$ \\
\hline Evaluación & $\begin{array}{l}\text { Disminuyó } \\
\text { Se mantuvo igual } \\
\text { Aumentó } \\
\text { Nunca hubo }\end{array}$ & $\begin{array}{r}109 \\
289 \\
15 \\
14\end{array}$ & $\begin{array}{r}25,5 \\
67,6 \\
3,5 \\
3,2\end{array}$ \\
\hline $\begin{array}{l}\text { Número de Anestesiólogos } \\
\text { por Quirófano }\end{array}$ & $\begin{array}{l}\text { Disminuyó } \\
\text { Se mantuvo igual } \\
\text { Aumentó } \\
\text { Nunca hubo }\end{array}$ & $\begin{array}{r}191 \\
206 \\
25 \\
5\end{array}$ & $\begin{array}{r}44,7 \\
48,2 \\
5,8 \\
1,1\end{array}$ \\
\hline $\begin{array}{l}\text { Número de Médicos Resi- } \\
\text { dentes por Quirófano }\end{array}$ & $\begin{array}{l}\text { Disminuyó } \\
\text { Se mantuvo igual } \\
\text { Aumentó } \\
\text { Nunca hubo }\end{array}$ & $\begin{array}{r}242 \\
134 \\
50 \\
1\end{array}$ & $\begin{array}{r}56,6 \\
31,3 \\
11,7 \\
0,2\end{array}$ \\
\hline
\end{tabular}

las actividades que debieron realizar durante la pandemia. No realizaron actividades sin la protección adecuada pero debieron llevar adelante actividades distintas a las de su programa. Se sintieron acompa- ñados por sus asociaciones de anestesiología (y por los responsables de sus instituciones) y volverían a elegir la especialidad. La pandemia afectó la vida personal de los residentes, quienes coinciden en haberse 
Tabla 3. Características de las actividades educativas y asistenciales en la residencia

\begin{tabular}{lcc}
\multicolumn{1}{c}{$\mathbf{n}=\mathbf{4 2 7}$} & $\mathbf{S i}$ & $\%$ \\
Se organizó un sistema de equipos de trabajo para las tareas asistenciales & 342 & 80 \\
Atendió pacientes con COVID-19 positivo & 362 & 84,7 \\
Se contagió COVID-19 como producto de su práctica profesional & 60 & 14 \\
Tuvo que realizar aislamiento & 309 & 46,3 \\
Tuvo compañeros de residencia con COVID-19 positivo & 65 & 72,3 \\
Algún familiar suyo se contagió de usted COVID-19 & 60 & 15,2 \\
Algún familiar suyo realizó aislamiento por su contacto con usted & 18 & 14 \\
Estuvo de licencia por presentar factores de riesgo & 335 & 72 \\
Siente que su formación se vio perjudicada durante la pandemia & 78,4 \\
\hline
\end{tabular}

sentido más estresados, haber experimentado episodios de ansiedad, tristeza y depresión, y haber tenido trastornos del sueño o dificultades para dormir. No refieren haber aumentado el consumo de alcohol $u$ otras sustancias, como puede verse en la Tabla 4.

Las principales preocupaciones de los residentes vinculadas a la pandemia se vincularon a la organización del sistema de salud y de la práctica asistencial, la afectación psicológica, el miedo a la enfermedad, el contagio y la muerte; y las consecuencias sobre la formación.

Del total de residentes, 175 (40,9\%) manifestó preocupaciones vinculadas a la Organización del sistema de salud y de la práctica asistencial (sobrecarga de trabajo, mala remuneración, mala organización, falta de insumos, multiempleo, falta de protocolos),

"falta de entrenamiento y recursos humanos" "sobrecarga de trabajo"

"abuso del recurso que representa el residente" "colapso del sistema de salud"

"precarización laboral" "falta de personal ante el aumento de casos, falta de educación con respecto a los nuevos protocolos" "Y que nos dejen de explotar y nos den la ayuda con insumos y personal que merecemos." "Mucho del equipo epp me lo compré con mi dinero" "Me preocupa la sobreexigencia que tenemos, la posibilidad de cumplir roles para los cuales no tenemos la mejor capacitación (asistencia en UTI")

"Falta de epp, falta de acompañamiento, hacinamiento, desorganización, cambio de funciones, no hay medidas claras, cambio de directivas día a día".

Por otro lado, 97 residentes $(22,7 \%)$ señalaron preocupaciones vinculadas a su Afectación psicológica (tristeza, angustia, estrés, enojo, incertidumbre).

"Preocupación por mis familiares y mi pareja, incomodidad para trabajar, incertidumbre sobre el futu- ro, ansiedad por que se resuelva" "Estrés, desvalorización, angustia, enojo" "abandono" "incertidumbre constante" "incertidumbre, tristeza, burn out, cansancio, soledad".

Además, 130 residentes $(30,44 \%)$ señaló preocupaciones vinculadas al Miedo a la enfermedad, el contagio y la muerte propio y de los seres cercanos

"miedo de contagiar a mis familiares" "La salud de mi familia, ser vector de la enfermedad para personas de riesgo" "Riesgo de vida para mi, para mi familia y la gente que me rodea".

Finalmente, 75 residentes $(17,56 \%)$ mencionaron como preocupaciones vinculadas a la pandemia las Consecuencias sobre la formación (Prácticas, rotaciones, políticas de ingresos de nuevos residentes).

"No aprendí lo necesario por esta pandemia" "Espero que se solucione lo antes posible la pandemia para mejorar mi formación" "Siento que me han puesto a cumplir con tareas para las cuales no estoy capacitado. Y mi formación como anestesiólogo se ha visto muy afectada" "falta de educación con respecto a los nuevos protocolos" "disminución de actividades prácticas, ingreso de nuevos residentes" "Formación suspendida pero no así la cantidad de guardias ni disminución a personal mínimo indispensable. Ahora ingresan nuevos q no podremos formar y será aglutinación de gente innecesariamente" "suspensión de la formación"

"Muy pocos procedimientos para muchos residentes." "Número de residentes y residentes nuevos, formación, supervisión".

La nube de palabras (Figura 1a) mostró las preocupaciones con respecto a la formación durante este período, vinculadas fundamentalmente a la disminución de prácticas y el descenso del número de procedimientos, la disminución de la calidad educativa y 


\begin{tabular}{|c|c|c|}
\hline En mi institución recibí información periódica sobre la pandemia & 3,53 & 1,28 \\
\hline Me capacitaron en el uso de EPP & 3,95 & 1,24 \\
\hline Me capacitaron en protocolos de procedimientos & 3,84 & 1,23 \\
\hline Me siento suficientemente capacitado para atender pacientes COVID & 3,85 & 1,06 \\
\hline La supervisión de mis actividades (asistenciales) se mantuvo durante la pandemia & 3,74 & 1,29 \\
\hline Me siento capacitado para todas las actividades que tuve que realizar & 4,01 & 1,02 \\
\hline Realice actividades en otras funciones a las que determinaba mi programa & 2,92 & 1,62 \\
\hline Realicé actividades asistenciales sin la protección recomendada & 2,14 & 1,32 \\
\hline Me sentí acompañado por los responsables de mi programa & 3,58 & 1,31 \\
\hline Me sentí acompañado por los responsables de mi institución & 2,95 & 1,42 \\
\hline Me sentí acompañado por mi Asociación de Anestesiología & 4,05 & 1,11 \\
\hline Volvería a elegir la especialidad Anestesiología & 4,72 & 0,76 \\
\hline Me sentí más estresado de lo habitual durante la pandemia & 4,02 & 1,22 \\
\hline He experimentado episodios de ansiedad a partir de la pandemia & 3,17 & 1,49 \\
\hline He experimentado episodios de tristeza o depresión a partir de la pandemia & 3,1 & 1,49 \\
\hline He comenzado o aumentado la ingesta de psicofármacos (ansiolíticos, etc.) & 1,36 & 0,95 \\
\hline He comenzado o aumentado el consumo de alcohol & 1,74 & 1,14 \\
\hline He comenzado o aumentado el consumo de alguna droga no legal & 1,18 & 0,66 \\
\hline He comenzado o aumentado consultas con algún profesional de salud mental & 1,79 & 1,34 \\
\hline He presentado dificultades para dormir o trastornos del sueño & 2,78 & 1,52 \\
\hline
\end{tabular}

Totalmente en desacuerdo $=1$ y Totalmente de acuerdo $=5$.
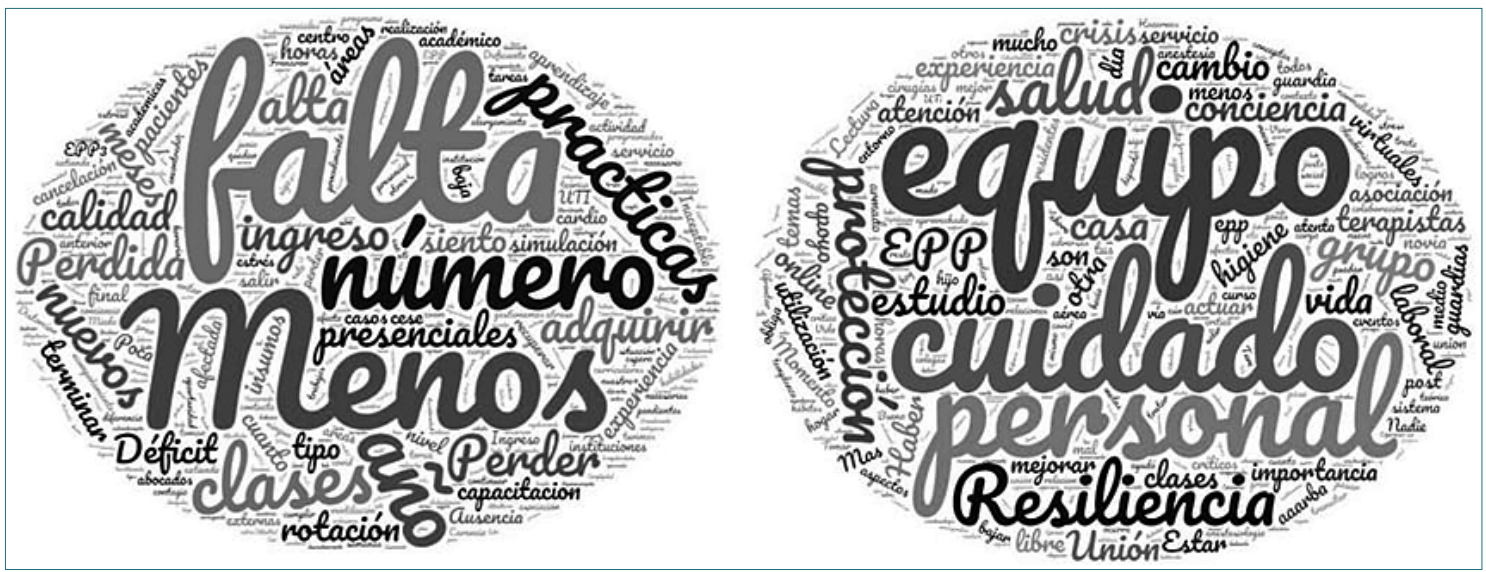

Figura 1. Nube de palabras que refleja las preocupaciones con respecto a la formación durante la pandemia (a) y a los aspectos positivos de haber transitado esta experiencia (b).

la pérdida de clases y otras oportunidades de aprendizaje). Por otro lado, los residentes mencionaron aspectos que pueden considerar positivos de haber atravesado la pandemia, como se observa en la nube de palabras (Figura 1b), entre ellos mayor énfasis en el cuidado personal, el equipo, desarrollo de resiliencia. 
Treinta del total de los residentes encuestados manifestaron no reconocer ningún aspecto positivo.

\section{Discusión}

Nuestro estudio mostró que la mayoría de los residentes de anestesiología percibió que su formación estuvo afectada por la pandemia. La mayoría de las provincias y distritos estuvieron representados en la muestra (18/19 distritos en los que existen programas de residencia de anestesiología y el 50\% correspondió a la provincia de Buenos Aires y Ciudad Autónoma de Buenos Aires. Esta distribución centralizada es consistente con la distribución poblacional de la Argentina y sus espacios de formación[13]. Asimismo, el 90\% de las residencias fueron de gestión pública al igual que los registros del Ministerio de Salud de la Nación[14]. El $55 \%$ de nuestra muestra fue de género masculino, si bien la feminización de los egresados de la carrera de medicina es de 70\%[15] la elección de la especialidad es todavía masculina. La muestra se obtuvo durante las semanas epidemiológicas 39 y 40, luego 196 días de aislamiento social preventivo obligatorio en la que el registro de contagiados y muertos por COVID-19 en la Argentina era de 751.001 casos acumulados, 16.937 fallecidos acumulado y 14.392 nuevos casos al día[16].

La mayoría de los residentes refirió que su formación se vio afectada durante la pandemia y las de gestión pública en mayor medida. Es indudable el brutal impacto que la pandemia tuvo en todos los niveles de la vida ciudadana. El sistema sanitario fue el epicentro donde se desarrollaron las consecuencias de la pandemia y quien debió implementar significativas adaptaciones para asistir a la población. Indefectiblemente, los programas con formación en servicio en salud fueron afectados en mayor medida.

El mayor impacto educativo se manifestó en el descenso en el número de procedimientos, rotaciones programáticas, clases presenciales y actividades de simulación. Estos datos son similares a los publicados en las residencias de anestesiología del resto del mundo[8],[9],[10]. Llamativamente, se observó poco uso de la simulación, a pesar de la gran difusión de esta herramienta educativa en la enseñanza de la anestesiología en el mundo[17] y de estar incluido como una recomendación de formación por parte de la Federación Argentina de Asociaciones de Anestesia, Analgesia y Reanimación (FAAAR)[18] y del Ministerio de salud de la Nación en el marco de referencia para la formación en residencias de la especialidad Anestesiología en nuestro país[14]. La telemedicina, muy difundida por la pérdida de presencialidad durante pan- demia[19] también fue poco frecuente. La simulación y telemedicina se presentan como oportunidades de mejora en los programas vigentes de anestesiología.

En los resultado surgió la posibilidad de aprendizajes de nuevas habilidades, como ser el desempeño en situaciones de crisis o el énfasis en el trabajo en equipo y el cuidado personal, tal cual se menciona en editoriales que reflexionan sobre la experiencia de haber transitado una pandemia para los anestesiólogos[20] y la posibilidad de que la pandemia aparece como una oportunidad de repensar la formación en residencias[21]. La incorporación de estos nuevos aprendizajes a futuro implica reformular los programas de las residencias.

La mayoría de los residentes refirió que se organizaron equipos de trabajo para las tareas asistenciales y se capacitaron para la atención de pacientes COVID-19. Sin embargo, el $14 \%$ se contagió como producto de su práctica profesional, la mitad debió realizar aislamiento y $72 \%$ tuvo compañeros enfermos por COVID-19. Seguramente se contagiaron en contextos sociales, ya que en las semanas epidemiológicas en las cuáles se realizó la encuesta el pico epidémico estaba en pleno desarrollo. La población que mayormente ha incumplido la recomendación de no participar de reuniones sociales han sido los jóvenes. No ha sido indagado específicamente el grado de cumplimiento, pero, se podría inferir que los residentes pertenecen a este grupo.

Las instituciones desarrollaron protocolos principalmente en los quirófanos y salas de internación. Otros espacios asistenciales y educativos no fueron protocolizados y esto podría explicar la cantidad de profesionales afectados con COVID-19.

La pandemia afectó en forma significativa la vida personal de los residentes, quienes manifestaron haberse sentido más estresados, haber experimentado episodios de ansiedad, tristeza y depresión. Estos resultados coinciden con las publicaciones sobre la afectación del bienestar de los profesionales de la salud en la pandemia y los residentes en particular[5]. Manifestaron preocupación por el contagio de seres queridos, por la muerte; y por la organización del sistema de salud con sobrecarga laboral y escasez de protocolos.

Afortunadamente, en los últimos años, los sistemas de residencia, además de garantizar el aprendizaje y disminuir el error médico procuran que los ambientes en que se desarrolla el aprendizaje ocurra en un buen clima de aprendizaje asegurando el bienestar de los residentes. La pandemia afectó todos los sistemas de cuidado, tanto el de los pacientes cómo los de los integrantes del sistema de salud. Los responsables de los sistemas de residencia deberán velar 
por estos valores.

A pesar de que la mayoría de los residentes percibió que su formación fue afectada por la pandemia y del negativo impacto en su vida personal, una enorme cantidad seguiría eligiendo la especialidad.

Este estudio tiene varias limitaciones, se realizó a través de una encuesta electrónica enviada por la Federación Argentina de Asociaciones de Anestesia, Analgesia y Reanimación (FAAAR), algunos residentes podrían haberse sentido condicionados en sus respuestas o no contestar. No todas las provincias estuvieron representadas y contestaron un poco más de la mitad de los residentes. Si bien este trabajo refleja la realidad de un sólo país, la globalización y el nivel de conexión en el que vivimos permitió que una infección originada en China rápidamente llegara al resto del mundo, constituyéndose en pandemia. La realidad y el contexto de cada país, sus sistemas de salud y educativos, llevan a que la afectación de la formación sea distinta, pero las coincidencias con otras publicaciones muestran que hay puntos de encuentro y que compartir información y experiencias, haciendo el esfuerzo de adaptarla a cada realidad nos permite capitalizar esta experiencia. Consideramos que es un estudio valioso que muestra la realidad de las residencias de anestesia en la Argentina durante la pandemia y pone en evidencia una situación que requiere planificar acciones para remediar este déficit en la formación en este período.

\section{Conclusión}

La mayoría de los residentes de anestesiología percibió que su formación estuvo afectada por la pandemia y muchos se vieron afectados emocionalmente. La formación se vio comprometida por la disminución del número de prácticas y procedimientos, la suspensión de las rotaciones, actividades académicas presenciales y por el hecho de que los residentes debieron dejar de desempeñarse en actividades de su especialidad y dedicarse a la asistencia en respuesta a la necesidad asistencial de la pandemia. Los residentes transitaron este período con estrés, angustia, incertidumbre y alteraciones del sueño.

La presente investigación no ha recibido ayudas específicas provenientes de agencias del sector público, sector comercial o entidades sin ánimo de lucro.

\section{Referencias}

1. Andrew K. Hall, Markku T. Nousiainen, Paolo Campisi, J. Damon Dagnone, Jason R. Frank, Karen I. Kroeker, Stacey Brzezina, Eve Purdy \& Anna Oswald (2020) Training disrupted: Practical tips for supporting competency-based medical education during the COVID-19 pandemic, Medical Tear, 42:7, 756- https://doi.org/10.1080/014 2159X.2020.1766669

2. Tolu LB, Feyissa GT, Ezeh A, Gudu W. Managing Resident Workforce and Residency Training During COVID-19 Pandemic: Scoping Review of Adaptive Approaches. Adv Med Educ Pract. 2020;11:527-535 https:// doi.org/10.2147/AMEP.S262369

3. Zoia C, Raffa G, Somma T, Della Pepa GM, La Rocca G, Zoli M, et al. COVID-19 and neurosurgical training and education: an Italian perspective. Acta Neurochir (Wien). 2020;162(8):1789-94. https://doi.org/10.1007/s00701020-04460-0

4. Baptista, Fabíola Vieira Duarte, Aguiar, Marilia Ribeiro de Azevedo, Moreira, Joanne Alves, Sousa, Felipe Carvalho Barros, Plenns, Glauco Cabral Marinho, Simao, Raif Restivo, Ruffini, Vitor Maia Teles, Lin, Chin An, \& Nunes, Maria do Patrocínio Tenório. (2020). Contributions of residents from multiple specializations in managing the COVID-19 pandemic in the largest public hospital Brazil. Clinics, 75, e2229. Epub August 19, 2020. https://doi.org/10.6061/ clinics/2020/e2229

5. Khusid JA, Weinstein CS, Becerra AZ, Kashani M, Robins DJ, Fink LE, et al. Well-being and education of urology residents during the COVID-19 pandemic: Results of an American National Survey. Int J Clin Pract. 2020;74(9):e13559. https://doi. org/10.1111/ijcp.13559

6. Clark VE. Impact of COVID-19 on neurosurgery resident research training. J. Neurosurg. 2020:1-2. https://doi. org/10.3171/2020.4.JNS201034

7. Ferrario L, Maffioli A, Bondurri AA, Guerci C, Lazzarin F, Danelli P. COVID-19 and surgical training in Italy: residents and young consultants perspectives from the battlefield. Am. J. Surg. 2020 https://doi.org/10.1016/j. amjsurg.2020.05.036

8. Sneyd, J. R., Mathoulin, S. E., O'Sullivan, E. P., So, V. C., Roberts, F. R., Paul, A. A., Cortinez, L. I., Ampofo, R. S., Miller, C. J., \& Balkisson, M. A. (2020). Impact of the COVID-19 pandemic on anaesthesia trai- 
nees and their training. British journal of anaesthesia, S00070912(20)30570-5. Advance online publication. https://doi. org/10.1016/j.bja.2020.07.011

9. Hong-Fei Zhang, Lulong Bo, Yun Lin, Feng-Xian Li, Shujun Sun, Hong-Bin Lin, Shi-Yuan $\mathrm{Xu}$, Jinjun Bian, Shanglong Yao, Xiangdong Chen, Lingzhong Meng, Xiaoming Deng; Response of Chinese Anesthesiologists to the COVID-19 Outbreak. Anesthesiology 2020; 132:13331338 https://doi.org/10.1097/ ALN.0000000000003300

10. Haldar R, Kannaujia AK, Shamim R, Dongare $P$, Mondal $H$, Agarwal A. A national survey evaluating the effect of COVID-19 pandemic on the teaching and training of anaesthesiology postgraduate students in India. Indian J Anaesth 2020;64:227- https:// doi.org/10.4103/ija.IJA_645_20

11. El-Boghdadly, K., Wong, D.J.N., Owen, R., Neuman, M.D., Pocock, S., Carlisle, J.B., Johnstone, C., Andruszkiewicz, P., Baker, P.A., Biccard, B.M., Bryson, G.L., Chan, M.T.V., Cheng, M.H., Chin, K.J., Coburn, M., Jonsson Fagerlund, M., Myatra, S.N., Myles, P.S., O'Sullivan, E., Pasin, L., Shamim, F., van Klei, W.A. and Ahmad, I. (2020), Risks to healthcare workers following tracheal intubation of patients with COVID-19: a prospective international multicentre cohort study. Anaesthesia, 75: 14371447. https://doi.org/10.1111/ anae. 15170

12. Lee, J., Chan, J., Ithnin, F., Goy,
R., \& Sng, B. L. (2020). Resilience of the restructured obstetric anaesthesia training programme during the COVID-19 outbreak in Singapore. International journal of obstetric anesthesia, 43, 89-90. https://doi.org/10.1016/j. ijoa.2020.04.002

13. Duré, I., Cadile M. , (2015) "La gestión de recursos humanos en salud en Argentina: una estrategia de consensos". Ciudad Autónoma de Buenos Aires. Ministerio de Salud de la Nación. Dirección Nacional de Capital Humano y Salud Ocupacional. Disponible en: https://www. academia.edu/20126075/La_gestión_de_Recursos_Humanos_de_ Salud_en_Argentina_Una_estrategia_de_consensos_2008_2015

14. Ministerio de Salud y Desarrollo Social de la Nación "Marco de Referencia para la formación en residencias del equipo de salud: Especialidad: Anestesiología. Disponible en: https://www. argentina.gob.ar/sites/default/ files/anestesiologia_-_documento_marco.pdf

15. Borracci, Raúl A., Salazar, Adriana I., \& Arribalzaga, Eduardo B. (2018). El futuro de la feminización de la medicina en Argentina. FEM: Revista de la Fundación Educación Médica, 21(3), 113-118. https:// doi.org/10.33588/fem.213.944

16. Informes diarios Ministerio de Salud de la Nación Argentina. Disponible en https://www.argentina.gob.ar/informes-diarios/ septiembre-de-2020

17. Bhaskar, S., Bradley, S., Chattu, V. K., Adisesh, A., Nurtazina, A., Kyrykbayeva, S., Sakhamuri, S.,
Yaya, S., Sunil, T., Thomas, P., Mucci, V., Moguilner, S., IsraelKorn, S., Alacapa, J., Mishra, A., Pandya, S., Schroeder, S., Atreja, A., Banach, M., \& Ray, D. (2020). Telemedicine Across the Globe-Position Paper From the COVID-19 Pandemic Health System Resilience PROGRAM (REPROGRAM) International Consortium (Part 1). Frontiers in public health, 8, 556720 . https://doi.org/10.3389/ fpubh. 2020.556720

18. "Normas o condiciones mínimas para la residencia de anestesiología" Resolución de la Federación Argentina de Asociaciones de Anestesia, Analgesia y Reanimación (FAAAAR). Disponible en https://www.anestesia.org.ar/assets/downloads/articles/129/125normas_residencias.pdf

19. Ena J. (2020). Telemedicine for COVID-19. Telemedicina aplicada a COVID-19. Revista clinica espanola, 220(8), 501502. https://doi.org/10.1016/j. rce.2020.06.002

20. Ferrando C, Colomina MJ, Errando CL, Llau JV. Anesthesiology and the Anesthesiologists at COVID-19. Anestesiología y los anestesiólogos en la COVID-19. Rev Esp Anestesiol Reanim. 2020;67(6):289-291. https://doi. org/10.1016/j.redar.2020.05.005

21. Goldhamer MEJ, Pusic MV, Co JPT, Weinstein DF. Can Covid Catalyze an Educational Transformation? Competency-Based Advancement in a Crisis. New England Journal of Medicine. 2020;383(11):1003-5. https:// doi.org/10.1056/NEJMp2018570 